\title{
Development and current status of treatment for pediatric indirect inguinal hernia
}

\author{
Mo Zhifeng ${ }^{1}$,Tan Yuanfei ${ }^{1}$,He Hanzhong ${ }^{2}$, Zhang Zhuorong ${ }^{2}$, Yang Wenbin ${ }^{1 \#}$ \\ ${ }^{1}$ Emergency and Disaster Medical Center, the seventh Affiliated Hospital of Sun Yat-Sen University, Shenzhen 518000 , \\ China; Mo Zhifeng, E-mail:edifen@126.com,https://orcid.org/0000-0001-9238-2242. \\ ${ }^{2}$ Department of Pediatri Surgery, Guangzhou Women And Children's Medical Center, Guangzhou 510000, China; \\ ${ }^{\#}$ Yang Wenbin is the corresponding author, Email 13829282179@139.com, https://orcid.org/0000-0002-5850-9374.
}

Article History Received 5 March 2020 Accepted 25 April 2020 Published 30 June 2020

Cite this Article Mo Zhifeng, Tan Yuanfei, He Hanzhong, Zhang Zhuorong, Yang Wenbin. Development and current status of treatment for pediatric indirect inguinal hernia [J].Medical Research, 2020.2(2):1-8, http://dx.doi.org/10.6913/MRHK.202006_2(2).0001

Copyright (C) 2020 Creative Publishing Co., Limited. All rights reserved. Email:mrhk26640333@gmail.com.

\begin{abstract}
To investigate the development and current status of the treatment of pediatric indirect inguinal hernia. Inguinal hernias include indirect hernias and direct inguinal hernias, but there are almost indirect inguinal hernias in children. Pediatric indirect inguinal hernia that the most common disease in pediatric surgery is almost caused by patent processus vaginali which incidence ranges from $0.8 \%$ to $4.4 \%$. The treatment of pediatric indirect inguinal hernia reflects the process of pediatric surgery development, which from conservative non-surgical treatment to high ligation of the longitudinal incision hernia sac. In recent years, with the continuous improvement of medical level in China, laparoscopic technique has been applied in hospitals all over China, and laparoscopic high ligation of indirect inguinal hernia sac has become the standard for the treatment of pediatric indirect inguinal hernia.
\end{abstract}

Key Words Inguinal hernia; Treatment; Infants; Children; Laparoscopic Surgery

\section{Surgical anatomy of the groin in children}

The anatomy of groin in children: In the early stages of embryogenesis, its peritoneum protrudes down from the deep inguinal ring to form the processus vaginalis, which extends downward along the gubernaculum testis as the testis descends and merges into the scrotum. Initially, the testis is at the level of 2nd-3rd lumbar vertebrae in the retroperitoneum ${ }^{[1]}$, as the embryo grows, its testis gradually descends, and the processus vaginalis closes off after the testis enters the scrotum when the fetus is about 8 months. In this stage, the blind pouch of the processus vaginalis wraps the testis, but the canal of processus vaginalis on the upper part of the testis still communicates with the abdominal cavity. Therefore, the incidence of indirect inguinal hernia in premature infants is much higher than that in full-term infants. The incidence of indirect inguinal hernia in children is $0.8 \%-4.4 \%{ }^{[2]}$, however, the documented incidence in premature infants is as high as $16 \%$ $25 \%^{[3-5]}$. After 8 months till the birth of the fetus, the closure process of the peritoneal processus vaginalis follows a certain order, in which the deep inguinal ring closes off first, followed by the upper part of the testis, and finally the spermatic cord. Meanwhile, the processus vaginalis is left in the testis and forms the proper 
Mo Zhifeng, Tan Yuanfei, et al. Development and current status of treatment for pediatric indirect inguinal hernia Medical Research ISSN 2664-0333 eISSN 2664-0341 Volume 2 Issue 2 http://dx.doi.org/10.6913/MRHK.202006_2(2).0001

tunica vaginalis cavity of the testis, which no longer communicates with the abdominal cavity ${ }^{[6]}$. However, the closure of the peritoneal processus vaginalis can be uncompleted, stopped, or delayed due to various factors, thus causes a permanent complete opening or a partial opening. If it is a complete opening, the hernia contents can protrude into the scrotum, causing a testicular hernia; if it is a partial opening, the contents can protrude into the groin, causing a spermatic cord hernia. Unclosed processus vaginalis is the main cause of indirect inguinal hernia in children and also the anatomical basis for the formation of this disease ${ }^{[7]}$. When a baby patient's abdominal pressure increases due to crying, its organs in the abdominal cavity protrude into the processus vaginalis to form hernia contents. Studies have shown that the right processus vaginalis develops a little bit later than the left one, so about $60 \%$ of indirect inguinal hernias in children occur on the right side ${ }^{[8]}$. We found in clinical surgeries that most of children with indirect inguinal hernia also have problems in testicular descent, which may indicate that the closure of peritoneal processus vaginalis is closely related to the process of testicular descent ${ }^{[9]}$. Some researchers speculated that this is related to the role of male hormones. Hutson et al. found that the reduction of calcitonin-related protein secreted by the genitofemoral nerve can result in the complete opening or partial opening of the processus vaginalis. However, no androgen receptor was found in the processus vaginalis, so further study of its specific mechanism needs to be done ${ }^{[10]}$.

\section{Conservative treatment of indirect inguinal hernia in children}

Studies have shown a possibility that the peritoneal processus vaginalis would close 6 months after birth ${ }^{[11]}$, which is rare in clinical practice and possibly caused by the frequent crying of children. The abdominal organs of children, such as the small intestine and the ovaries, would partially protrude into the processus vaginalis with increased abdominal pressure. It will have a certain impact on the peritoneal processus vaginalis even if no indirect inguinal hernia is formed; and a small amount of the abdominal effusion will flow into the processus vaginalis, which would also be one of the reasons why the processus vaginalis is rarely closed after the birth of children. Many family members have concerns when getting conservative treatment for infants, especially premature babies. Transient conservative treatment is feasible when the family members of the children are fully informed of the risks and closely followed up for observation. A few scholars believe that for the children with occult indirect inguinal hernia, the possibility of getting overt indirect inguinal hernia in the children can be reduced by observing and waiting. Based on related literature references, there are three main methods for conservative treatment of indirect inguinal hernia.

\subsection{Hernia band therapy}

Hernia band therapy is mainly to compress the internal ring orifice of indirect inguinal hernia with foreign objects so that the intra-abdominal organs no longer protrude into the peritoneal processus vaginalis, giving the processus vaginalis an opportunity of self-healing to achieve the therapeutic effect. There are specialized hernia bands used clinically and self-made ones created by individual scholars. Babies have tender skin and are more prone to swelling and rupture. Cotton pads should be applied to strengthen and protect the skin at the groin no matter which type of hernia band is applied to infants within 6 months. Parents are hard to master the bandaging method of hernia bands and require the guidance or even participation from professional medical staff during the replacement process, which is why this treatment is rarely promoted. The hernia band therapy does not achieve the therapeutic effect if the band is tied too loose and can lead to congestion and varicocele of the spermatic vein if the band ties too tight. It has not been reported in relevant literature whether the hernia band will affect the blood supply of the testis in the long run leading to testicular atrophy and thus affecting the reproductive function of children. Theoretically, short-term application and appropriate tightness of the hernia band do not lead to changes in testicular blood supply; having a too-tight hernia band will inevitably cause crying and restlessness in children, so it is particularly important to educate children's families about the proper tightness of the hernia bands to achieve early awareness and adjustment. Academician Zhang Jinzhe 
Mo Zhifeng, Tan Yuanfei, et al. Development and current status of treatment for pediatric indirect inguinal hernia Medical Research ISSN 2664-0333 eISSN 2664-0341 Volume 2 Issue 2 http://dx.doi.org/10.6913/MRHK.202006_2(2).0001

${ }^{[12]}$ believes that hernia band treatment for the children with an indirect inguinal hernia can only be used transiently or in combination with other methods.

\subsection{Injection therapy}

Injection therapy is to create adhesions around the hernia sac and in combination with local compression to achieve the purpose of treatment. Aseptic inflammation around the peritoneal processus vaginalis happens through injecting sclerosing agents such as Absolute Alcohol, Sodium Morrhuate, Glycerin Tartrate, glycerin alum, or 50\% glucose around the hernia sac. Sometimes multiple injections and compression are required to get better results. In the early 20th century, injection therapy was very popular in Europe and the United States, and a few scholars in China brought up this method. However, this method is highly possible to cause injuries of the spermatic artery, spermatic vein and vas deferens, forming an inguinal or scrotal hematoma, leading to the adhesion of vas deferens and spermatic vessels in the long run, which affects the blood supply of testis and leading to testicular atrophy; besides, it would cause an intestinal adhesion, intestinal necrosis and even peritonitis due to the blindness of injection and likelihood of sclerosing agent flowing into the abdominal cavity ${ }^{[13]}$. This method has been scarcely applied by scholars in the field of professional pediatric surgery due to its uncertain long-term efficacy, many complications, and high risks.

\subsection{Traditional Chinese Medicine Therapy}

Traditional Chinese medicine therapy on the treatment of children with indirect inguinal hernia has been rarely reported in China recently and is usually used after surgical treatment based on previous documentations. He Zemin ${ }^{[14,15]}$, Yu Cuimin ${ }^{[16]}$, etc. used traditional Chinese medicine with a local external application, Pan Guoyuan ${ }^{[17]}$ used traditional Chinese medicine with acupuncture, Qian Lifang ${ }^{[18]}$ used massage with an external application, etc., which are reported to have a certain effect. However, it is not mentioned in the field of professional pediatric surgery or even in pediatric surgery textbooks. At present, further research and data are needed to support the therapeutic effect and we should have an open attitude.

\section{Surgical Treatment of Indirect Inguinal Hernia in Children}

The surgical methods of indirect inguinal hernia in children are aiming at the internal ring. The high ligation of the internal ring can be performed without repairing and strengthening the anterior and posterior walls. It all reflects the progress and development of pediatric surgery from early longitudinal incision to transverse abdominal incision, and from three-port laparoscopy to single-port laparoscopy.

The high ligation of indirect inguinal hernia sac in children can be divided into traditional trans inguinal surgery and laparoscopic surgery. The traditional trans inguinal surgery is subdivided into the early longitudinal incision of external oblique muscle diaphragm and abdominal transverse line minimally invasive surgery without incision of external oblique muscle diaphragm; laparoscopic techniques carried out in China for the treatment of indirect inguinal hernia in children are double-port laparoscopic technique, single-site twoport laparoscopic technique. The single-port laparoscopic technique is subdivided into simple internal ring suture, internal ring suture ligation of the transected hernia sac, the addition of medial umbilical ligament to cover the internal ring, high ligation of extraperitoneal indirect inguinal hernia sac, extraperitoneal water injection to separate the internal ring ligation, double ligation of the internal ring, etc. ${ }^{[19-23]}$. All surgical procedures have a common feature, dealing with the internal ring. Most of the pediatric surgery scholars believe that although the peritoneal processus vaginalis is possible to close after the birth of a child, indirect inguinal hernia rarely heals spontaneously, so surgery is recommended as soon as diagnosed ${ }^{[24]}$. Infants have a high chance of getting indirect inguinal hernia incarceration due to frequent crying, increased intra-abdominal pressure, and family members lacking medical knowledge or other reasons. The risk of incarceration in premature infants is $30 \%{ }^{[25]}$. Related documentations show that infants with a history of incarceration have a risk of re-incarceration of $15 \%{ }^{[26]}$ within 5 days. For immature newborns, surgery can be considered as long 
Mo Zhifeng, Tan Yuanfei, et al. Development and current status of treatment for pediatric indirect inguinal hernia Medical Research ISSN 2664-0333 eISSN 2664-0341 Volume 2 Issue 2 http://dx.doi.org/10.6913/MRHK.202006_2(2).0001

as their weight is more than $2 \mathrm{~kg}$.

\subsection{Open Surgery}

The traditional high ligation of the indirect inguinal hernia sac is the most classical standard procedure for the treatment of children with indirect inguinal hernia. Adult surgeons play the role of pediatric surgeons in early times, and they selected a procedure of incising external oblique muscle sac using abdominal longitudinal incision, separating the hernia sac, and ligating the processus vaginalis. With the further comprehension of indirect inguinal hernia in children, the development of pediatric surgery, and the establishment of specialized children's hospitals in each province and city, the surgical procedure for indirect inguinal hernia in children at present is using a small transverse abdominal incision (about $1 \mathrm{~cm}$ ), which does not require an incision of the external oblique muscle diaphragm but only raises the spermatic cord from the external ring orifice, separates the hernia sac, and ligates the processus vaginalis ${ }^{[27]}$.

Open surgery was the most classical operation before laparoscopic surgery becomes popular. The advantage is that it does not require general anesthesia with endotracheal intubation but only caudal anesthesia in children and the results meet expectations, however, limitations exist. First, occult hernias cannot be detected or explored during surgery, only indirect inguinal hernia can be treated. It has been reported in the literature that the detection rate of occult hernia by preoperative inguinal color Doppler ultrasound before surgery is as high as $90 \%{ }^{[38]}$, but the child has to take the risk of a second operation when missed diagnosis. Second, open surgery causes greater damage to the inguinal area tissue. The spermatic vessels and vas deferens are separated during high ligation of the peritoneal processus vaginalis, which breaks the original anatomical structure of the inguinal region and may damage the vas deferens and spermatic vessels, affect testicular blood supply, cause testicular atrophy and even lead to reproductive dysfunction.

\subsection{Laparoscopic Surgery}

With the development of clinical medicine and growing numbers of pediatric surgery specialized hospitals, laparoscopic treatment of indirect inguinal hernia in children has been quite popular. Laparoscopic high ligation of indirect inguinal hernia sac has been developed with a variety of methods from using three holes, two holes to a single hole; there are complete extraperitoneal ligation of the processus vaginalis and also intraperitoneal suture of the processus vaginalis ${ }^{[28,29]}$. The choice of surgical method depends not only on the type of indirect inguinal hernia but also on the skills and experience of the surgeon in laparoscopic surgical procedures. Safe and effective ligation of the peritoneal processus vaginalis through minimizing the abdominal wall operating hole, reducing damage to the surrounding hernia sac tissue, and avoiding injury to the vas deferens and spermatic vessels has become the consensus for the treatment of indirect inguinal hernia in children and reducing postoperative recurrence.

\subsubsection{Laparoscopic-assisted High Ligation of the Extraperitoneal Indirect Inguinal Hernia Sac}

A 5-mm trocar was placed at the umbilicus, and another trocar was placed at the right abdominal wall or para-umbilicus. High ligation of the processus vaginalis is usually accomplished using homemade hernia needles, epidural puncture needles, or endoclose needles. Most importantly, a trocar was placed at the same time at the umbilicus and beside the umbilicus, so single-site single-port laparoscopic surgery was performed to reduce the injury of the abdominal wall and achieve the purpose of minimally invasive surgery. Laparoscopic-assisted high ligation of extraperitoneal indirect inguinal hernia sac includes lift-and-insert internal ring ligation, double-hole snare suture internal ring ligation, and forceps needle-assisted internal ring ligation. Zhang Liang ${ }^{[30]}$ and Jiang Qing ${ }^{[31]}$ each reviewed and analyzed 212 cases and 316 cases of indirect inguinal hernia in children treated with laparoscopic-assisted lifting and inserting internal ring ligation, which showed less intraoperative bleeding, no damages on the surrounding organs, and good postoperative results. Wang Jun and Zhou Xin et al. ${ }^{[32]}$ used transumbilical double-hole snare suture to ligate the internal ring in the 
Mo Zhifeng, Tan Yuanfei, et al. Development and current status of treatment for pediatric indirect inguinal hernia Medical Research ISSN 2664-0333 eISSN 2664-0341 Volume 2 Issue 2 http://dx.doi.org/10.6913/MRHK.202006_2(2).0001

treatment of 750 cases of indirect inguinal hernia in children. There were 6 cases of postoperative recurrence of indirect inguinal hernia. The recurrence rate was $0.8 \%$, which is significantly lower than the $2 \%-4 \%$ of traditional surgery. Liu Sujun and Wang Fan et al. ${ }^{[33]}$ used laparoscopic forceps needle-assisted internal ring ligation in the treatment of 106 cases of indirect inguinal hernia in children. 21 cases of occult hernia were found during the operation, and the incidence rate is $19.8 \%$. The child could walk and be discharged on the same day after the operation. There was no recurrence or related complications during follow-up within the next 1-4 months.

\subsubsection{Laparoscopic transabdominal ring suture ligation}

It is not necessary to incise in the groin area using this method, but at least two or three operation holes are required to complete the operation. At present, the most widely used operation is single-site two-hole surgery through the umbilicus. It is divided into simple internal ring suture ligation and internal ring suture ligation transecting the hernia sac. Simple internal ring suture ligation only requires purse-string suture or continuous suture of the internal ring of the hernia, while the internal ring suture of the transected hernia sac requires the surgeon to incise the peritoneum circumferentially in the neck of the hernia sac, precisely separate the vas deferens and spermatic vessels, transect the hernia sac and followed by purse-string suture or continuous suture of the internal ring. Shi Qunfeng et al. ${ }^{[34]}$ used two-port laparoscopic internal ring suture ligation in the treatment of 1586 cases of indirect inguinal hernia in children. The operations were successful. There was no conversion to laparotomy during the operation. There were 5 cases of postoperative recurrence, and the recurrence rate is $0.3 \%$. No intestinal adhesion, intestinal obstruction, incisional hernia, testicular atrophy, etc were reported in these children within the 3-5 years of follow up. Silk Hongwei et al. ${ }^{[35]}$ treated 1536 children with an indirect inguinal hernia by single-site single-hand laparoscopic internal ring suture ligation at umbilicus without any intraoperative intestinal injury or converting to laparotomy. There were 5 cases of postoperative recurrence, and the recurrence rate is $0.32 \%$. Garcia-Hernandez, C et al. ${ }^{[36]}$, and Boo, $\mathrm{Y} \mathrm{J}$ et al. ${ }^{[37]}$ respectively achieved good results in treating 285 children and 202 children through laparoscopic internal ring suture ligation of the transected hernia sac. However, at present, pediatric surgeons believe that high ligation of the hernia sac in children can be performed simply without transacting the hernia sac. The more increasing excessive abdominal operation, the greater risk of the surgery and children, moreover, it is rarely reported in China of this operation due to its high requirements and needing precise skills for the surgeon.

\section{Advantages and disadvantages of laparoscopic surgery and discussions of related problems}

Laparoscopic treatment of indirect inguinal hernia in children continues to be completely impossible to avoid the damage of vas deferens and spermatic vessels in children; there will be postoperative knot reaction, scrotal edema, testicular atrophy, etc. therefore, it is impossible to completely avoid the recurrence of indirect inguinal hernia. In addition to all surgery-related complications related to surgical methods, the experience of surgeons and the maturity of techniques also account for a large proportion. However, at present, most scholars believe that it has many advantages such as less trauma, shorter hospitalization, and faster postoperative recovery. With the rapid development of medical technology, the application of laparoscopy is becoming more and more popular, and there are increasing reports on the laparoscopic treatment of indirect inguinal hernia in children in the field of pediatric surgery domestic and international. Laparoscopic high ligation of indirect inguinal hernia sac has become the standard treatment for indirect inguinal hernia in children and has been unanimously recognized by experts in the field of pediatric surgery around the world.

The advantages of laparoscopic treatment of indirect inguinal hernia in children are summarized as follows: (1) It can treat bilateral inguinal lesions at the same time, including occult hernia, communicating hydrocele, and so on. It cannot be surpassed by traditional trans inguinal surgery that a single incision completes the ligation of the internal ring at one time. (2) It can minimize the changes in inguinal anatomy and 
Mo Zhifeng, Tan Yuanfei, et al. Development and current status of treatment for pediatric indirect inguinal hernia Medical Research ISSN 2664-0333 eISSN 2664-0341 Volume 2 Issue 2 http://dx.doi.org/10.6913/MRHK.202006_2(2).0001

avoid damages to the vas deferens and spermatic vessels; It has a unique advantage because it is not necessary to dissect the structure of the inguinal canal during surgery or separate the vas deferens and spermatic vessels to achieve high ligation of the internal ring. (3) It can examine and treat other abdominal organ diseases with a single incision.

The application of laparoscopy in pediatric surgery and even the whole field of surgery is an inevitable trend, but there are several problems worth to consider: (1) It takes time to accumulate operation skills for each pediatric surgeon to master laparoscopy from supporting the lens, punching operation holes, knotting, etc.. It is crucial for the occurrence of intraoperative complications and postoperative recovery that the operator accumulates experience and proficiency. Literally, it is not a minimally invasive surgery because the wound of the abdominal wall in children is small rather the intra-abdominal injury is large. Children, in the period of growth, has especially relatively delicate organs, therefore, it is a requirement for every pediatric surgeon to be cautious and careful. (2) Does it have to choose laparoscopic surgery to be minimally invasive if the older child has been diagnosed with only a unilateral indirect inguinal hernia for many years? Combined with my clinical experience, it is feasible for the child's family members to choose as long as alternative surgical plans are patiently and thoroughly illustrated. It is reasonable to choose Trans inguinal surgery for older children with only unilateral indirect inguinal hernia, and it must combine the surgeon's skills and proficiency to select a minimally invasive surgery method. (3) It is probably because of the short-time existing of laparoscopic surgery and the difficulty of long-term following up for children that it is rarely reported that the long-term vas deferens obstruction effects from Trans inguinal surgery and laparoscopic surgery for indirect inguinal hernia in children. Although it involves dissecting the tissue around the hernia sac and separating the vas deferens and the spermatic vessels in trans inguinal surgery, it can straighten out the vas deferens without angulations and distortion; however, because of the absolute high ligation of the internal ring in laparoscopic surgery, it is more likely to cause vas deferens distortion during the hernia needle passing through the vas deferens and spermatic vessels, and angulation during lifting and knotting. It has not been reported in relevant literature whether laparoscopic surgery will lead to vas deferens obstruction in children in the long run. The author believes that it may have a less significant impact on the children with unilateral indirect inguinal hernia, but we need to consider the possibility of related complications in the children who undergo laparoscopic high ligation of both sides of the indirect inguinal hernia sac.

\section{ACKNOWLEDGEMENT}

This study was supported by Guangzhou Women And Children's Medical Center and the Seventh Affiliated Hospital, Sun Yat-sen University.

The research was performed in the Seventh Affiliated Hospital, Sun Yat-sen University.

\section{STATEMENT}

There is no conflict of interest in this article.

\section{REFERENCES}

[1] Li Xiaojun, Liu Jialin, Bi Jiangang, et al. Application of medical umbilical ligament flap covering the internal ring to reinforce laparoscopic herniorrhaphy in children and teenagers with indirect inguinal hernias [J]. Chinese Journal of Hernia and Abdominal Wall Surgery (Electronic Edition), 2012, 6 (3): 59-61.

[2] Mo Zhifeng, Li Xiaowei, Lu Yingchou, et al. Clinical application of laparoscopic double purse-string plus suture with medical umbilical flap high ligation of internal ring in the treatment of giant inguinal hernia in children [J]. Journal of Clinical Pediatric Surgery, 2015, 0 (2): 133-135. 
Mo Zhifeng, Tan Yuanfei, et al. Development and current status of treatment for pediatric indirect inguinal hernia Medical Research ISSN 2664-0333 eISSN 2664-0341 Volume 2 Issue 2 http://dx.doi.org/10.6913/MRHK.202006_2(2).0001

[3] Galinier P, Bouali O, Juricic M, et al. [Focusing of inguinal hernia in children][J]. Arch Pediatr, 2007,14(4):399-403.

[4] Gulack B C, Greenberg R, Clark R H, et al. A multi-institution analysis of predictors of timing of inguinal hernia repair among premature infants[J]. J Pediatr Surg, 2018,53(4):784-788.

[5] Weaver K L, Poola A S, Gould J L, et al. The risk of developing a symptomatic inguinal hernia in children with an asymptomatic patent processus vaginalis[J]. J Pediatr Surg, 2017,52(1):60-64.

[6] Chen Kaiyun, Xiang Guo'an, Wang Hanning, et al. Laparoscopic high ligation of hernia sac and median umbilical fold covering internal ring in treating pediatric indirect inguinal hernia [J]. Chinese Journal Of Surgery, 2007,45(03):207-209.

[7] Zhang Yusong. Applied anatomy of the inguinal region in children [D]. Human Anatomy of Southern Medical University, 2011.

[8] Liu Qin, Yang Xiaohong, Chen Xinlin, et al. Prenatal diagnosis of ultrasound and MRI in the simple fetal indirect hernia [J]. Chinese Journal of Medical Ultrasound (Electronic Version), 2015,12(11):878-883.

[9] Liu Xiang, Peng Bo, Qu Han, et al. Clinical feasibility of modified Bevan and Saha-jeap in the treatment of high cryptorchidism with indirect inguinal hernia in children [J]. National Journal of Andrology, 2019,25(03):279-282.

[10] Clarnette T D, Hutson J M. The genitofemoral nerve may link testicular inguinoscrotal descent with congenital inguinal hernia[J]. Aust N Z J Surg, 1996,66(9):612-617.

[11] Cai Wei. Pediatric Surgery [M]. Fourth Edition. People's Medical Publishing House, 2014.

[12] Zhang Jinzhe. Acute Abdomen in Children [J]. Journal of Clinical Pediatric Surgery, 2007 (01): 60-63.

[13] Zhou Dekai, Tian Xiaofei, Lv Longzheng, et al. Inguinal Hernia Repair After Unappropriate Local Injection Therapy [J]. Chinese Journal Of Surgery, 1996(04):202-203.

[14] He Zemin. Clinical Observation on 62 Cases of Infantile Indirect Inguinal Hernia Treated by Yuqiao Decoction and Warming Traditional Chinese Medicine Sac Triangular Hernia Support [J]. Journal of Sichuan Traditional Chinese Medicine, 2006(06):73-74.

[15] He Zemin. Clinical Observation on 68 Patients of Lnguinoslanting Hernia Treated withthe Hernia[Wt4"Hz]Fcuring Drugs and Chinese Drugs Sac Fixed on the Focus through an Elastic Cord Net and Cloth Slices [J]. Chinese Journal of School Doctor, 2000(05):323-325.

[16] Yu Cuimin. Treatment of 20 Cases of Infantile Indirect Inguinal Hernia by External Application of Chinese Herbs [J]. Journal of Practical Traditional Chinese Internal Medicine, 1996(03):44.

[17] Pan Guoyuan. 36 Cases of Indirect Inguinal Hernia Treated with Acupuncture and Medicine [J]. Shanxi Journal of Traditional Chinese Medicine, 1999(02):24.

[18] Qian Lifang. 30 Cases of Infantile Inguinal Hernia Treated by Massage Combined with Hernia Band [J]. Massage and Guidance, 2003, 19 (5): 15.

[19] Shang Peizhong, Pan Hongxia, Wang Tieshan, et al. Current status and progress of laparoscopic surgery for indirect inguinal hernia in children [J]. Journal of Laparoscopic Surgery, 2018,23(11):868-871.

[20] Lv Qigang, Sun Xiaogang, Li Jinliang, et al. Comparison of the therapeutic effects of single-port and twoport laparoscope for inguinal hernia in children [J]. Journal of Shandong University (Health Science), 2018,56(02):51-55.

[21] Liu Xuelai, Zhang Chuang, Zhang Yongting, et al. Clinical application for single-port laparoscopic percutaneous extraperitoneal closure in the treatment of inguinal hernia in children [J]. Journal of Developmental Medicine(Electronic Version), 2018,6(02):77-81.

[22] Liu Jie, Duan Guangqi, Wang Xiao, et al. Coping skills for the adverse factors of laparoscopic percutaneous extraperitioneal closure of pediatric inguinal hernia [J]. Chinese Journal of Hernia and Abdominal Wall Surgery(Electronic Edition), 2017,11(01):44-45. 
Mo Zhifeng, Tan Yuanfei, et al. Development and current status of treatment for pediatric indirect inguinal hernia Medical Research ISSN 2664-0333 eISSN 2664-0341 Volume 2 Issue 2 http://dx.doi.org/10.6913/MRHK.202006_2(2).0001

[23] Li Yue, Li Shuhua. Application effect of single-hole endoscopic total extraperitoneal technique in pediatric inguinal hernia [J]. Journal of Modern Medicine \& Health, 2017,33(20):3168-3170.

[24] Shalaby R, Elsaied A, Shehata S, et al. Needlescopic assisted internal ring suturing; a novel application of low-cost home-made instruments for pediatric inguinal hernia repair[J]. Hernia, 2019.

[25] Gollu G, Ates U, Bahadir K, et al. Transinguinal laparoscopic evaluation of contralateral side during unilateral inguinal hernia repair for children[J]. J Pediatr Urol, 2019,15(5):561.

[26] Holcomb G R, Brock J R, Morgan W R. Laparoscopic evaluation for a contralateral patent processus vaginalis[J]. J Pediatr Surg, 1994,29(8):970-973, 974.

[27] Liu Ruyi, Xu Ke, Zhang Shiwei. Observation of curative effect of abdominal transverse cleavage line small incision on oblique inguinal hernia in children [J]. Chinese Journal of New Clinical Medicine, 2019,12(02):204-206.

[28] Li Solin, Xu Weili. Guidelines for laparoscopic surgery of inguinal hernia in children (2017 Edition) (previous section) [J]. Chinese Journal of Hernia and Abdominal Wall Surgery(Electronic Version), 2018,12(01):1-5.

[29] Li Solin, Xu Weili. Guidelines for Laparoscopic Surgery of Inguinal Hernia in Children (2017 Edition) (the next section) [J]. Chinese Journal of Hernia and Abdominal Wall Surgery(Electronic Version), 2018,12(02):81-85.

[30] Zhang Liang, Yang Jian, Zhang Wenyuan. High ligation of hernial sac in children assisted by minilaparoscope [J]. China Modern Doctor, 2010,48(20):157-158.

[31] Jiang Qing, Xiong Chaoxia. Operative cooperation of laparoscopic assisted lifting and inserting hernia sac high ligation in children[J]. Practical Clinical Medicine, 2011,12(11):102-103.

[32] Wang Jun, Zhou Xin, Luo Zhengli, et al. Laparoscopic treatment of indirect inguinal hernia in children by trans-umbilical dual-port methods, a report of 750 cases [J]. Chinese Journal of General Surgery, 2006(06):425-426.

[33] Liu Sujun, Wang Fan, Zhu Yilin, et al. Clamp type needle assisted single-hole laparoscopic treatment in pediatric patients with inguinal hernia [J]. Chinese Journal of Hernia and Abdominal Wall Surgery (Electronic Edition), 2014,8(01):16-18.

[34] Shi Qunfeng, Li Xinning, Luo Shuyou, et al. Laparoscopic transfixion of internal ring in treatment of oblique inguinal hernia for children [J]. Journal of Laparoscopic Surgery, 2010,15(11):847-849.

[35] Si Hongwei, Zhang Peng, Cui Qiangqiang, et al. Single-site laparoscopic closure of inner inguinal ring with single hand for pediatric inguinal hernias [J]. Journal of Clinical Pediatric Surgery, 2015(5):375-376, 382.

[36] Garcia-Hernandez C, Carvajal-Figueroa L, Suarez-Gutierrez R, et al. Laparoscopic approach for inguinal hernia in children: resection without suture[J]. J Pediatr Surg, 2012,47(11):2093-2095.

[37] Boo Y J, Han H J, Ji W B, et al. Laparoscopic hernia sac transaction and intracorporeal ligation show very low recurrence rate in pediatric inguinal hernia[J]. J Laparoendosc Adv Surg Tech a, 2012,22(7):720-723.

[38] Liu Wei. Laparoscopy combined with preoperative color Doppler ultrasound in the diagnosis and treatment of occult indirect hernia in children [D]. Shanxi Medical University, 2014. 